\title{
CORPOREIDAD Y LENGUAJE: LA ACCIÓN COMO TEXTO Y EXPRESIÓN
}

\section{BODYNESS AND LANGUAGE: THE ACTION AS TEXT AND UTTERANCE}

Dr. Sergio Toro-Arévalo (sergiotoro@uach.cl) Instituto de Filosofía y Estudios Educacionales. Universidad Austral de Chile (Valdivia, Chile).

\begin{abstract}
The present work has as objective to analyse the embodied dimension of language, reviewing the conceptual issues of cognition, mind and language. Starting from the emerging of new data and knowledge that come from different disciplinary areas and taking Merlau-Ponty's phenomenology as epistemological option, creating a critique to the functional approaches of language and its contrast with the mention author's descriptions and also the cognitive sciences. The results of the study are oriented to explicit and show the language as a process of vivid body or corporeal manifestation and presence, which establish the possibility of considering movement, understood as intentionality in movement, as a text or discursive nonverbal interpretation.
\end{abstract}

Key words: cognition, embodied language, epistemology, movement, phenomenology, action.

\section{Resumen}

El presente trabajo aborda un análisis sobre la dimensión encarnada del lenguaje, revisando los supuestos conceptuales de la cognición, mente y lenguaje. A partir de la emergencia de nuevos antecedentes y conocimientos provenientes de diferentes aéreas disciplinares y tomando la fenomenología de MerleauPonty como opción epistémica se realiza una crítica de las aproximaciones funcionales del lenguaje y su contraste con descripciones fenomenológicas y de las ciencias cognitivas. Los resultados del trabajo se orientan a explicitar el lenguaje como un proceso que expresa el cuerpo vivido o corporeidad, lo que establece la posibilidad de considerar la motricidad, entendida como intencionalidad en despliegue, como un texto o interpretación discursiva no verbal.

Palabras clave: Cognición, lenguaje encarnado, epistemología, motricidad, fenomenología, acción.

\section{Introducción}

En el presente trabajo (1) se pretende establecer desde el análisis de determinados pre-supuestos de la motricidad humana como condición y posibilidad del ser humano y, así mismo, como objeto de estudio científico y disciplinar y su relación con el lenguaje. Tal relación se plantea desde una aproximación básicamente fenomenológica en la visión de Merlau-Ponty, como sus continuadores (Thompson 2007, Noe 2004, Gallagher 2006, Gallagher y Zahavi 2008, Varela 2000), los cuales se pueden encontrar en disciplinas como la neurociencias, la filosofía, la psicología, sociología y la pedagogía. 
El propósito es aportar y profundizar en una comprensión del ser humano desde su acción y por consecuencia, siguiendo a Gadamer (1977) y Austin (1982), desde su lenguaje. En tal sentido cualquier análisis de la acción y del comportamiento humano es un análisis sobre la con-figuración y construcción de los procesos que le permiten expresar e im-primir su condición y posibilidad en conformidad con los sensus desde la existencia autónoma pero al mismo tiempo en un despliegue relacional.

La condición primera, no obstante se focaliza en aquello que podemos entender por humano en tanto ser y su eventual potencia de actuación. Es reconocido incluso más allá de los dos mil años de era cristiana, que la pregunta ontológica ha sido el gran impulso de la tradición filosófica griega hasta nuestros días, como también que dependiendo de la respuesta se pueden generar culturas y cosmovisiones que se pueden alzar como relatos de la presencia y sentido de lo humano.

Desde el punto de vista de la cultura cristiana occidental, la respuesta ha estado centrada en opciones que han abarcado todas las esferas del comportamiento y acción humana. Desde Platón, San Agustín y con la profundización y especificación racionalista y metodológica de Descartes (2), el entendimiento de un ser humano fragmentado y dividido en dos componentes, a saber, continente y contenido, forma y fondo, lo afectivo y lo efectivo, lo motor y lo sensible. Ello ha contribuido enormemente al estado actual del desarrollo de las ciencias, la sociedad, la tecnología y obviamente nuestra formas de relación cotidiana (Manuel Sergio 2007). La comprensión de una res extensa y una res cogitans ha marcado profundamente a occidente a niveles que se traducen en dualismos antropológicos y metodológicos que hoy en día se nos presentan con la emergencia desde el mismo devenir en tales campos de acción e interpretación, como una modificación y tensión o, en palabras de Bachelard, un obstáculo epistemológico (1997) que se adentra en un cuestionamiento de las bases epistemológicas y programáticas del dualismo cartesiano.

En resumen, el objetivo central que nos orienta es revelar la dimensión encarnada del lenguaje, desde el punto de vista epistemológico y eventuales implicancias en el análisis y despliegue en contextos disciplinares de las ciencias sociales.

\section{Cognición como procesamiento de información...la metáfora computacionalista}

Nos referimos explícitamente al desarrollo de una serie de teorías y posicionamientos científicos. Al mismo tiempo, el desarrollo de la tecnología ha marcado una era sin precedentes en la historia de la Humanidad. Tales avances han encontrado, no obstante, limitaciones y barreras no menores, sobre todo en aquellas que se focalizan en el abordaje del ser humano desde el punto de vista tecnológico, dado que no estaría siendo posible reducir ciertas manifestaciones de lo humano que permitan su homologación parcial y por lo menos de ciertas funciones específicas. El pensamiento cartesiano se ha visto aún más afectado por el desarrollo de una serie de teorías y posicionamientos científicos, programáticos, sociales y del último siglo como la física cuántica de Heinsemberg y Bohr (1981), la teoría del caos de Prigogine (1997) y el pensamiento complejo de Morin (2003), por nombrar algunos.

En este sentido, el campo de la investigación en la inteligencia artificial presenta un estado y desarrollo que ha alcanzado niveles de profundización y ampliación hacia áreas del conocimiento que hace algunos años se presentaban como ajenos o por lo menos lejanos a una operatividad mecanicista y funcional de los objetos de estudio particulares como, al mismo tiempo, de los posibles resultados de sus esfuerzos (Peifer y Bongard 2007). Con ello se han puesto en tensión sus procesos funcionales y sus matrices epistémicas, resultando una apertura hacia la lingüística, la filosofía y, como es claro, hacia vías alternativas dentro de las ciencias de 
la cognición y neurociencias que permitan mayor pertinencia y comprensión de lo humano, fundamentalmente en la relación estructura-acción-cultura.

Para efectos de la comprensión del contexto y en conformidad a (con) las intencionalidades del presente trabajo (3), realizaremos, en una primera instancia, una mirada general y breve de la evolución de dicho campo y su relación con la comprensión del ser humano y, en segundo lugar, con el lenguaje y la motricidad.

Comenzaremos, entonces, con la teoría de inteligencia, conocida también como la tesis computacional que comprende el proceso del pensamiento como un procesamiento o manipulación de símbolos discretos que se pueden ordenar y pre-configurar dependiendo de la rigurosidad en el respeto de la linealidad del proceso, a saber in-put, procesing y out-put, que sumado al proceso de retro-alimentación, se constituyó en lo que Wiener definió como modelo cibernético y que Von Neumann tradujo en lo que hoy conocemos como ordenador o computador digital. Aunque en la actualidad muchos de los modelos y tecnología han variado desde los años cuarenta del siglo pasado, básicamente se respeta la arquitectura inicial de su inventor.

Varela nos plantea que "se trató por cierto, de un decisivo avance tecnológico, pero su mayor importancia consistió en echar los cimientos para el enfoque dominante en el estudio científico de la mente, el cual cristalizaría en la siguiente etapa como el paradigma cognitivista" (1990:36) y en cuyo devenir fue traduciéndose en lo que se conoció como procesamiento simbólico o computacionismo. Enfatizamos que la tesis principal de esta corriente se traduce en sostener que el pensamiento es un procesamiento de representaciones simbólicas, las cuales, obviamente, en tanto símbolo debieran tener una dimensión física y semántica.

En tal sentido, un computador puede manipular y ordenar desde la correlación física de los símbolos, pero no tiene acceso a la dimensión semántica del mismo y, por otra parte una dimensión semántica podría tener o ser evocada desde diferentes dimensiones físicas o signos, por lo que se vuelve irreductible, no aprehensible y al mismo tiempo analógica y metafórica, dado que se adentra en la dimensión de la vivencia, afectiva y sensible.

Este programa ha tenido una gran aceptación y difusión dentro de la sociedad en sus diferentes niveles y campos de acción: por ejemplo, dentro de ciertos enfoques de la psicología cognitiva aplicados a la educación como el mismo aprendizaje significativo de Ausbel, el cual se expresa en una aproximación del conocimiento eminentemente lógico y digital, cuya solidificación y relación se estructura en base a correspondencias de informaciones previas con informaciones emergentes, donde los procesos analógicos o risomáticos son fundamentalmente distractores del hecho o conocimiento objetivo. Este enfoque se sostiene en una aproximación representacionista, cuya principio básico es que todo proceso significativo puede ser traducido en algoritmos o formalidades sintácticas y de igual forma sus eventuales aplicaciones en situaciones o problemáticas que obviamente han sido sometidas al mismo proceso (Thompson 2007).

El problema que genera este enfoque, se traduce en que durante la experiencia y vivencia de los procesos comunicativos y de conocimiento, los sujetos no comprenden ni interpretan de manera inequívoca e independiente de los procesos de contingencia, historicidad y lugar en los que se despliegan. Podríamos preguntarnos, en consecuencia, cómo y por qué influyen estos aspectos en los procesos de lenguaje y conversacionales; a qué nivel se producen las variaciones, singularidades y alteridades; cómo se relacionan 
la cognición con la experiencia subjetiva de cada sujeto; dónde se produce la diferencia y complementariedad entre cada cual en el caso que sean dos unidades distintas y cómo afectan al lenguaje.

Las preguntas podrían continuar, pero el hecho sustantivo es que la tesis representicionista simbólica, de acuerdo a lo descrito más arriba, no puede dar respuestas coherentes y satisfactorias. Esta conjetura dentro de las ciencias del conocimiento y neurociencias se ha denominado como el vacío explicativo (Thompson 2007, Varela 2000, Gallagher 2006, Noe 2004).

Este vacío se expresa en la funcionalidad y posibilidades que presentan los ordenadores y computadores personales, conocido como inteligencia artificial. Vale decir, como artefactos que nos presentan posibilidades de generación de unidades semánticas y semióticas propias, pertinentes a problemáticas o requerimientos que no fueron anticipados o pre-pro-gramados. De manera que la ampliación de facultades en tales sistemas es siempre heterónoma y alopoiética.

En caso contrario, si observamos el despliegue del lenguaje cotidiano dentro de los seres humanos pareciera que lo dicho adquiere otros elementos que radicalmente sobrepasan el uso de juegos matemáticos formales y predecibles, más bien, contemplan aspectos de incertidumbre, afecciones, variaciones orgánicas y funcionales y, por sobre todo modificación de lo comunicado y del mismo proceso de comunicación. Siempre pareciera existir un punto de fuga en el lenguaje que permite una ampliación e intensificación de los horizontes de sentido (Gadamer 1977) que los sistemas artificiales no logran conseguir.

En términos más específicos y funcionales, las unidades de condensación y recepción de información requieren de algunas propiedades que permitan la generación de otro tipo de información que al mismo tiempo no sólo se presenta como un algoritmo neutro; muy por el contrario, se presentan como sistemas que de alguna manera impactan y construye, sobre un proceso de captación y traducción de información, unidades de sentido que modifican tanto su comportamiento como su estructura. Además, dicho proceso se realiza en el ejercicio autónomo y autopoietico del sujeto como un todo y no como una unidad dividida en compartimientos periféricos y/o centrales.

En este sentido los enfoques cognocitivistas clásicos lentamente se fueron abriendo hacia el abanico de posibilidades que presentan las propiedades de los procesos biológicos y funcionales que determinan o, por lo menos condicionan, cualquier posibilidad de configuración del lenguaje, de modo tal, que la muy útil distinción entre software y hardware, propia del enfoque cognitivista clásico, en el caso de lo humano, resulta una falacia.

\section{Desde la falacia a la encarnación de la mente y del lenguaje}

La situación que se ha descrito más arriba constituye una herencia de una determina opción antropofilosófica que se ha desarrollado durante siglos en occidente apoyada tanto por su eficiencia dentro de grupos de interés y poder especifico como al mismo tiempo por contextos epistemológicos históricos acordes (4) que no se desarrollará en extenso en esta ocasión. Una visión alternativa o emergente requiere no sólo de ideas y supuestos alternativos, sino también de una modalidad diferente de presentación que permita explicar o posibilitar la comprensión. 
Válganos decir que intentamos abordar desde la complejidad misma del lenguaje y los sentidos, con sus analogías, su fugacidad, sus metáforas e irreductibilidad, una compresión de las emergentes propuestas del lenguaje, la acción y en definitiva del ser humano. Para tal empresa invoquemos a Mario Benedetti (1993) quien nos invita a danzar el siguiente vals:

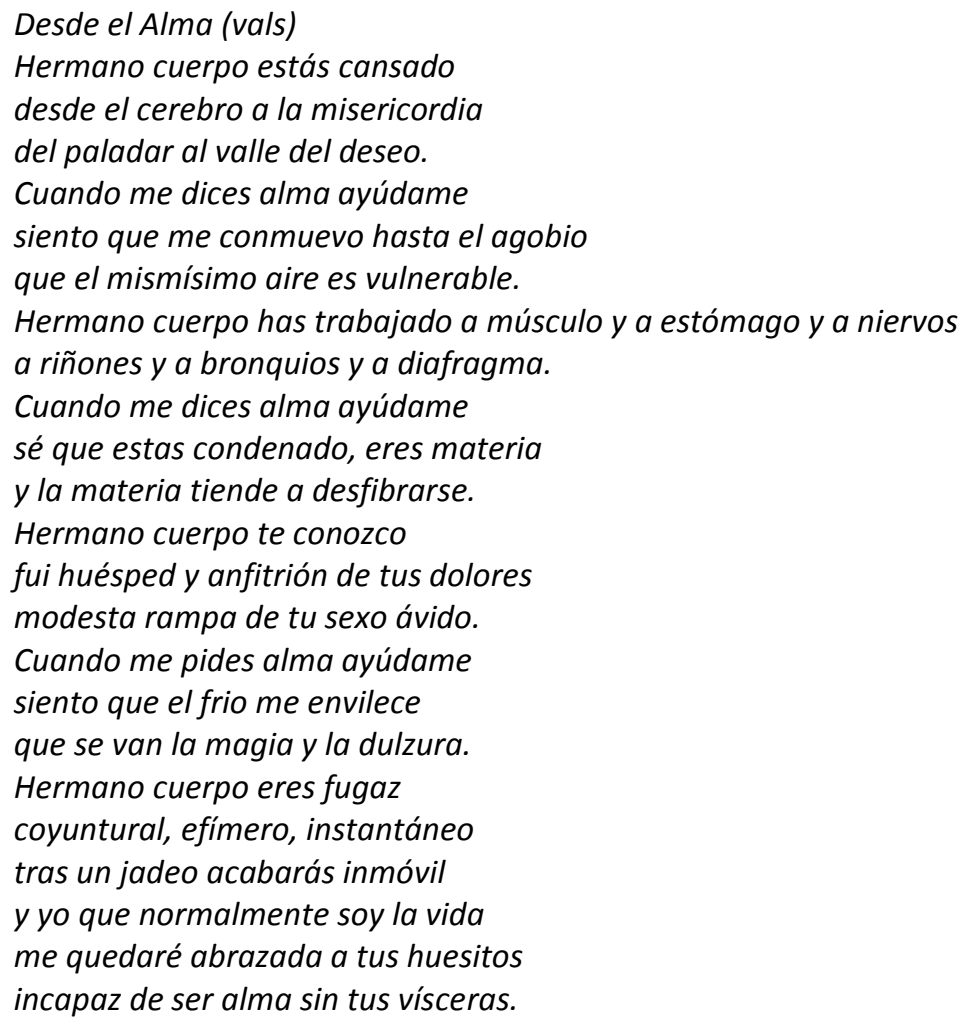

En este baile donde la distinción primera, expresada en el diálogo o por lo menos la voz de uno de ellos, lentamente se nos hace una segunda distinción que implica una unidad irrenunciable. Unidad que se sostiene en el ser, no el componente que no responde a una ontología diferente y autónoma que se genera en sí mismo, sino más bien que se produce en la relación. Desde un punto de vista fenomenológico sería intrascendente preguntarse qué es primero o cuál es el flujo de causalidad, pues la respuesta dependerá del lugar en que se ubique el observador.

Podemos reconocer, por lo tanto, varios elementos que identifican o caracterizan lo que denominamos, división alma y cuerpo, como el principio vital-sentiente del primero y el principio estructural-funcional del segundo. ¿Cómo podemos abordar esta relación sin caer en lo que criticamos del dualismo cartesiano; qué sería lo particular de un relato diferente desde la fenomenología?

Como no tiene sentido abordar esa teoría, desde la parte o distinción particular de alma y/o cuerpo, debemos comenzar por otra distinción que es mayor y posibilita la comprensión de lo que es un vals y como se despliega, a saber la acción. Acción o vivencia que ocurre en un contexto, en un marco de relaciones que es posible identificar sin desnaturalizar el baile o su gestualidad, que ocurre en tanto se produce la musicalidad y diálogo de los danzantes. 
Tal situación implica un grado de conocimiento de qué se hace, dónde se está y con quién; no obstante, el aspecto radical, el para qué, el propósito o intencionalidad de la acción es sólo distinguible como una emergencia de los factores o cuestionamientos anteriores (Husserl 1949). Pero lejos de configurar una intencionalidad como predeterminación, implica como una orientación, estar dirigido, o volcado hacia, como contenido de subjetividad y conciencia de la misma. Pero cómo es que se produce tal proceso en una forma no mediada a priori por una entidad metafísica o desencarnada.

Las características de la encarnación de la mente o sus implicancias nos iluminan en este sentido (Pfeifer y Bongard 2007):

1. Cualquier sistema inteligente requiere o está constituido desde un sistema senso-motor visible y palpable que le permite un acoplamiento con el entorno como también la sensación o consciencia de sí mismo. Un entendimiento de este factor se refiere a los componentes sensitivos que se ubican desde lo celular a lo sistémico, en el caso de la piel, como estructura, a macro identidades como el sistema digestivo o locomotor. Todas las unidades o sistemas mencionados comparten componentes de sensibilidad interna como externa que permiten la percepción de un estado global o lo que se ha denominado como Self (Damasio 2000, Pascual 2009).

2. Muchas tareas cognitivas se tornan más fáciles en la medida que la encarnación se ve implicada o desplegada. Dado que la acción al ser directa sobre algo y en un determinado contexto permite mayores recursos sensitivos $\mathrm{y}$, por tanto, mayores posibilidades comprensivas.

3. La ubicación de los receptores de sensibilidad en cada organismo y/o robot define el tipo de percepción e inteligencia que se estructura en general y en particular, vale decir no da los mismo en qué lugar se encuentra la distribución de la sensibilidad y su posterior traducción en la representación y constitución interpretativa. Pues cualquier elemento se encuentra directamente relacionado con una multiplicidad de factores temporo-espaciales, de densidad, en relación a la tarea, a la biomecánica, entre otros. Y cualquier modificación, por mínima que sea, afecta a todos los demás en nivel sistémico y particular.

Es significativo, por lo tanto, preguntarnos cómo se pueden interpretar las características mencionadas desde un esfuerzo fenomenológico y hermenéutico.

El primer aspecto en este ejercicio interpretativo desde la fenomenología, se relaciona con el sentir o la dimensión sensoria o explorativa (Toro 2002) de la experiencia humana, vale decir, en cada acción que se emprende, se explora y se conoce el mundo independiente del sentido o direccionalidad de la misma acción, ya que cada instante del proceso de ejecución de la misma requiere una contrastación de la efectividad y de la amplitud, ritmo e intensidad de lo que se está ejecutando. Incluso si observamos la generación de la acción desde un punto de vista proyectivo, vale decir en una suerte de etapa de planificación u organización, las informaciones requeridas para ésta provienen de algún tipo de afección o sentir del sí mismo y del entorno inmediato. Vale decir, una acción no se produce como un corolario de procesos sucesivos, desprendidos y relacionados desde una producción en línea, sino en un proceso integrado que al mismo tiempo es afectivo y efectivo. Por lo menos a la experiencia humana le es negada la posibilidad de distinción entre lo sensitivo y la actuación. Por el contrario, emerge como un mismo proceso y fenómeno. 
Desde este proceso primario se producen o generan las otras dimensiones de la acción, tales como lo constructivo y lo representativo (Gabler 1996). Uno de los resultados irrenunciables de este aspecto, es el carácter expresivo de la acción, cuyo corolario y modalidad puede ser la palabra o el lenguaje escrito (Gallagher 2006).

Ahora bien, podemos cuestionarnos si la tematización del sentir, que procede de forma regresiva, permite responder de vuelta a la ambición que anima el término o signo, o sea fundar la posibilidad de la palabra como tal. ¿Cómo efectuar el paso del sentir, cuyo carácter de pertenencia es a la carne del mundo, a la palabra como producción de sentido, como virtualidad de la experiencia pone en evidencia? ¿Cómo establecer una continuidad entre el ser-ahí de la cosa, que manifiesta cierta positividad, y la evanescencia del sentido lingüístico? ¿Cómo pensar la diferencia de lo invisible enfrente a lo visible, diferencia que sostiene la posibilidad de la expresión, sin reducirlo a una entidad positiva? ¿En qué es "el mismo ser que percibe y que habla", y cuál es el ser de éste? En resumen, ¿en qué sentido la percepción puede ser calificada de logos silencioso, o de expresión primordial, según los términos del Lenguaje indirecto y las voces del silencio?

Merleau-Ponty (2000) parte desde la experiencia del tacto y de la reversibilidad, con el fin de deshacer las oposiciones del pensamiento objetivo: así, explicita la revisión ontológica que esta reversibilidad requiere antes que revelar la esencia del sentir. Ahora bien, la posibilidad de fundar la expresión lingüística en el silencio de la percepción, es decir pasar de un modo de proceder regresivo a un modo progresivo, requiere una reflexión aún más radical, orientada sobre la esencia misma del sentir. Podemos hoy día señalar que es en la motricidad donde se genera y realiza. Entiendo motricidad como la ex-presión e im-presión de la corporeidad en pos de la superación de la limitación, la personalización y la en-culturación (Toro 2006, Manuel Sergio 2007). En palabras de Merleau-Ponty la motricidad es intencionalidad operante, es un serpresente, encarnado, sensible y motor al mismo tiempo.

De esta manera, en el bucle carne y entorno-mundo es que se produce el proceso de lenguajear o el contexto de lo desarrollado hasta aquí en el actuar, por lo tanto debemos adentrarnos en la unidad de la percepción y el movimiento: en tal situación podemos situarnos en medida de inscribir en ello la palabra, sólo así se tematiza la percepción desde esta profundidad. Merleau-Ponty (2000) anota en efecto: "Para elucidar Wahrnehmen y Sich bewegen (5), mostrar que ningún Wahrnehmen no percibe a condición de ser Sí mismo de movimiento. Partir desde ahí se puede comprender el lenguaje como fundamento del yo pienso: el lenguaje es al yo pienso lo que es el movimiento a la percepción". Así, es posible contemplar la exigencia de pensar la experiencia en continuidad con la palabra, más allá de toda referencia a una positividad, factual o ideal, lo que lleva a Merleau-Ponty a comprenderla como praxis antes que como teoría: el espíritu salvaje, anota en varias ocasiones, es espíritu de praxis.

El análisis intelectualista, aquí como en todas partes es, más que falso, abstracto. La "función simbólica" o la "función de representación" subentiende nuestros movimientos, sí, pero no es un término último para el análisis, se apoya, a su vez, en un cierto suelo, y el error del intelectualismo consiste en hacerla apoyar en sí misma, en separarla de los materiales en los que se realiza... se abstiene expresamente de introducir tras la palabra, la acción y la percepción, una conciencia simbólica que sería la forma común y numéricamente una de los materiales lingüísticos, perceptivos y motores (Merleau-Ponty 2000: 141). 
Tal planteamiento no sólo significa que el sujeto percibiente deba ser un sujeto motor, sino que la constitución de la cosa no puede ser cumplida sin referencia a la cinestesia. Tal es la perspectiva de Husserl (1949) de Ding und Raum en Ideen II. En este texto, distingue, en efecto, dos tipos de sensaciones que tienen funciones distintas. Por una parte, sensaciones que, por las aprehensiones que le son concebidas, constituyen en esbozos los rasgos correspondientes de la cosa como tal; por otra parte, sensaciones que no son objeto de tales aprehensiones sino que, además, son percibidores necesariamente de todas las aprehensiones de este tipo relativo a otras sensaciones, en la medida que las motivan, y que forman parte del objeto de una aprehensión de un todo de otro tipo que así pertenece como correlato a toda aprehensión constituyente. En otros términos, a la cinestesia no le corresponde ningún rasgo cualitativo del objeto y sin embargo participan a su constitución y son, por ende, animados por una aprehensión: motivan la aparición del objeto a tal distancia, según tal orientación. Si bien es cierto que cada cualidad posee una extensión que le es propia, es sobre todo en la cinestesia que descansa la constitución de la cosa propiamente tal como lo idéntico de todas las apariciones posibles: la amplitud y variabilidad de estos posibles remite en efecto a la libertad cinestésica. Es precisamente a esta distinción que Merleau-Ponty aporta, según su punto de vista, con el concepto de motricidad esencial del sujeto que hace estallar el marco de un análisis husserliano cuestionando la originalidad del datum sensible. Señala, por ejemplo, a propósito del tacto, que la encarnación o Leib (cuerpo vivido), no es una suma de tocarse (de " sensaciones táctiles "), ni tampoco una suma de sensaciones táctiles + cinestesia, sino más bien es un yo puedo. La fórmula que evocábamos al comienzo debe entonces ser entendida en un sentido fuerte: el percibir sólo se comprende desde la motricidad, la verdad del Wahrnehmen reside en el Sich bewegen, la conciencia perceptiva es originariamente conciencia motricia.

¿Qué significa Sich bewegen (movimentar-se)? Que el cuerpo se mueva no significa que una conciencia impulse un movimiento y que éste se despliegue en la extensión geométrica: El movimiento, señala Merleau-Ponty (2000), no es una decisión de espíritu, un hacer absoluto, que decretaría, del fondo del retiro subjetivo, algún cambio de lugar milagrosamente ejecutado en la extensión. El sí mismo no es exterior al movimiento sino que al contrario, pasa en él: es como sí mismo que se mueve. Entonces sale de sí mismo, se trasciende activamente, y cabalga el espacio: el Sich bewegen es marcha antes que representación y lo hace, precisamente, es volcando por el lado de lo que hace parecer que la conciencia preserva la trascendencia; debido a que es atravesada por ese movimiento, la intencionalidad perceptiva es ciertamente apertura efectiva y activa al objeto antes que representación. Merleau-Ponty (2000) señala, en efecto, que en el decir que se tiene una vista de un objeto (el texto escrito por ejemplo), es decir que, el percibiente (comúnmente llamado observador), va ya de sí a él, ya que ciertamente sale de sí en él. Esto precisa por tanto, que en la percepción, es el propio cuerpo o corporeidad el que se sumerge hacia una profundidad que en ningún caso es sólo geométrica sino también semántica y pragmática.

Sin embargo, no podemos quedarnos ahí. Si bien es cierto que el sí mismo es su propio movimiento, pasa en él, lo que no quiere decir que ese movimiento sea un movimiento de un sí mismo, lo que impediría su identificación con un desplazamiento objetivo. Concebir ese movimiento de esta manera resultaría restaurar una conciencia ubicua delante la cual éste se desplegaría. Se reactiva, entonces, la dualidad de un cuerpoobjeto y conciencia desencarnada. Así, en la medida que la conciencia es intrínsecamente móvil, su movimiento no podría ser confundido con un desplazamiento en el espacio geométrico. Es decir, hay una diferencia esencial entre el movimiento de las cosas y él del cuerpo fenomenal: "Llamo a una cosa que es movida, pero mi cuerpo, él, se mueve, mi movimiento se despliega y repliega. No es en la ignorancia de sí mismo, no es ciego para sí mismo, sino que irradia de un sí mismo, desde una autorrefencia en 
desenvolvimiento". Si el sí mismo pasa en un movimiento y se aleja en el mundo, se reencuentra él mismo en esa superación, de modo que ese alejamiento es sin distancia. Por consiguiente, Merleau-Ponty (2000) señala que mi movimiento vivido como tal, permanece invisible como movimiento objetivo: lo que se opone a lo que yo me veo es un invisible de hecho primero (mis ojos son invisibles para mí) pero, más allá de ese invisible (cuya cavidad se colma por el otro y mi generalidad) es un invisible de derecho: no puedo verme en movimiento, asistir a mi movimiento. Ahora bien, ese invisible de derecho significa en realidad que Wahrnehmen y Sich bewegen son sinónimos. El sí mismo pasa en la exterioridad, pero ésta permanece suya y entonces deber ser distinguida de la exterioridad objetiva: ella es, precisamente, exterioridad intencional.

Con todo, decir que hay un sí mismo de movimiento, implica reconocer que existe una conciencia que anima la motricidad, y ésta es un modo de conciencia más profunda que la recepción o la posesión de una cualidad; razón por la cual la cinestesia no es sólo una sensación que indica un carácter espacial: es una manera de llevarse hacia el objeto. Merleau-Ponty señala en diversas instancias que la percepción visual supone un movimiento de los ojos, tal como un enfoque, tanto así que en el descubrimiento de las cualidades táctiles del objeto, que imprimo con mi mano la velocidad y la presión necesarias. Entonces, hay que admitir que el movimiento sabe las cosas antes de saberlas, que la visión se precede en él. En cuanto el Sich bewegen se distingue del movimiento objetivo, volviéndose así sinónimo de Wahrnehmen, dado que la transcendencia de la cosa se distingue de la exterioridad objetiva, entonces, puede fenomenalizarse. El análisis de la motricidad permite evidenciar un sentido nuevo de la fenomenalidad: en la medida que el movimiento emana de un sí mismo, hay que admitir una percepción que le es propia, algo como un parecer motor. Esta conclusión ya está presente en la Fenomenología de la percepción (Merleau-Ponty 2000), cuyos análisis consagrados a la sensación desembocan sobre la idea de una "praktognosis", es decir, de un conocimiento práctico, el cual debiera ser reconocido como original y quizás como originario.

En efecto, la vuelta por la psicología permite evidenciar una significación motora de cualidades sensibles, en particular, el color conseguido a través de una actitud del cuerpo fenomenal antes que por una conciencia representativa: en lugar de ser un espectáculo objetivo, la cualidad se deja reconocer por un tipo de comportamiento que la pretende en su esencia y por eso en cuanto mi cuerpo adopta la actitud del azul obtengo una quasi-presencia del azul. Sin embargo, desde Merleau-Ponty (2000) la significación motora de los colores sólo se entiende si dejan de ser estados cerrados sobre ellos mismos o cualidades indescriptibles ofrecidas a la constatación de un sujeto pensante, y si por otra parte la motricidad deja de ser la simple conciencia de mis cambios de lugar presentes o próximos, podemos adentrarnos en una aproximación más sustantiva de la motricidad y su valencia en la configuración de sentido e interpretación, de lugar, habitabilidad y constitución de mundo. Es, por lo tanto, desde un mismo movimiento que la motricidad se define como una intencionalidad original antes que como la conciencia de un desplazamiento, y la sensación deja de aparecer como un contenido irreductible: el descubrimiento del sujeto motor permite revelar, sin llegar a la cualidad sensible, una significación motora. Ahora bien, eso es precisamente lo que falta en Husserl en el análisis evocado más arriba: el rechazo del carácter exponente de la cinestesia, entonces reducido a la conciencia de un cambio de lugar, el cual es llamado por las posiciones previas de data sensibles aprehendidos como cualidades del objeto. Si Husserl toma acta de sensaciones cenestésicas, su análisis sigue, no obstante, estructurado por la oposición a la cantidad y a la cualidad, del objeto y del sujeto, del movimiento corporal, del movimiento como globalidad temporo-espacial y de los registros vividos que lo señalan. Entonces de ello se desprende que la falta de intencionalidad motora es a la vez por exceso, en la medida que de la cualidad sensible sigue para él un contenido irreductible, que se generan por 
defecto como sensaciones de un desplazamiento, por lo que lo kinestésico estaría desprovisto de una pretensión significante que le sea propia.

Al contrario, según Merleau-Ponty, el sentido originario de la intencionalidad reside en este Sich bewegen que no es ni conciencia, ni movimiento. La conciencia en tanto conocimiento y el movimiento, en el sentido de desplazamiento en el espacio objetivo, son dos aspectos abstractos de una existencia. En efecto, el movimiento no se opone al sentir sólo si es concebido como un cambio de lugar y, el sentir no se opone al movimiento, sólo si es comprendido como posesión de una cualidad. La conciencia no es otra que su propio movimiento, pero éste no es una caída en la exterioridad: la conciencia no se echa fuera de sí mismo sólo para volver a encontrarse. El movimiento del sujeto es igualmente distancia y proximidad de sí mismo, un movimiento en el estado naciente, siempre comenzado y jamás desplegado, es dinamismo sin desplazamiento. El Sich bewegen, en consecuencia es ex e in-presión de la propia tensión interna evocada en la relacionalidad con el mundo, que en su proceso de despliegue, se constituye en identidad de entrar en sí mismo y del salir de sí mismo. Así, la intencionalidad bien puede caracterizarse como un "yo puedo" (según la propia expresión de Husserl), a condición de comprender esta potencia en el doble sentido de la potencialidad y de su puesta en marcha: siempre ya cumplida y siempre por cumplir, escapándose, por lo tanto, a la partición aristotélica del acto y su potencia.

\section{Lenguaje encarnado}

Este bucle en que están insertos la percepción y del movimiento, involucra la identidad de un ser y, por lo tanto, de su conciencia. De manera que al movimentar-se (sich bewegen) éste explaya e imprime su conciencia, su conocimiento y su posibilidades futuras de conocimiento. De ahí que la motricidad sea el sentido de todas las significaciones, no sólo desde el punto de vista funcional sino, fundamentalmente, desde el punto de vista emocional, afectivo y simbólico.

Por ende, dentro del plano de la construcción de la virtualidad de su experiencia -vale decir, de su lenguajeéste inevitablemente se genera desde la encarnación, desde la aprehensión del mundo, a través de la percepción y movimiento (Lakoff y Johnson 1999). De tal forma que el lenguaje siempre será una metáfora de la acción y de la experiencia en sí.

Esto trae, siguiendo a Lakoff y Johnson (1999), las siguientes consecuencias:

a. Dado que la mente es una encarnación, pretender una razón universal es, entonces, una quimera, más que una utopía. Es posicionamiento autónomo desde la experiencia, de despliegue y relacional en el sentido del lenguaje, por tanto histórica y situacional. Vale decir, cada persona desde su corporeidad o encarnación define su experiencia e identidad en un permanente acoplamiento con su entorno. Acoplamiento que al ser también estructural, hace imposible la existencia de una razón universal que defina una realidad positiva u objetiva. Razón por la cual no puede trascender su basamento que es al mismo tiempo su dinámica.

b. De lo anterior se desprende que la razón como proceso no sería consciente, toda vez que los mecanismos que permiten el razonamiento no están a completa disposición de la voluntad del sujeto (propioceptores y procesos neuromusculares, por ejemplo), sin embargo, la razón no es absolutamente no consciente (la metacognición es la expresión de tal situación), pero si el proceso de obtención o producción de la razón, vale decir, el razonamiento. 
c. En consecuencia, la razón no puede ser literal, desapasionada y precisa, objetiva y positiva. Sino más bien es analógica, simbólica y esencialmente emotiva. Por tanto ésta se caracteriza por ser fundamentalmente relacional-metafórica y su principal materia prima, el lenguaje, también lo es.

Estos tres aspectos tienen una repercusión enorme en lo que hoy intentamos definir como Motricidad Humana. Sobre todo desde el punto de vista de las posibilidades de estudios y de los campos de acción; por ejemplo, si consideramos el primer punto, surge inmediatamente la pregunta: ¿qué conocemos acerca de los pormenores de la corporeidad y, por tanto de la construcción del conocimiento? Al decir pormenores nos referimos a lo que la persona realiza día a día como una serie de acciones que, hasta el momento, sólo han sido tratadas y contempladas como triviales e insignificantes o desde un punto de vista morfo-funcional como: Comer, dormir, caminar, posiciones al sentarse y desplazarse, respirar, amplitud de acción y actitudes corpóreas, espacios relacionales y habitables, configuración geográfica, danza y juego, vestido y alimentación y su relación con la construcción del conocimiento, pero por sobre todo con el desarrollo humano. De la misma forma, no sabemos o poco nos hemos interesado, de la Motricidad Humana como un ejercicio de relación, como texto y red de sentidos en un contexto determinado. Pero al volver sobre este punto, es imposible evitar relacionar todo el comportamiento del ser humano, sobre todo aquello que Husserl (1949), Alfred Schütz (1993) y el propio Wittgenstein llamaron el Lebenswelt, es decir el mundo cotidiano, pues es ahí donde se construye lo que llamamos lenguaje en una contingencia permanente, recursiva pero no iterativa en una dinámica constante entre la encarnación-comportamiento-entorno. De tal manera que en la corporeidad se está en el mundo y en ella misma se cataliza el mundo y la persona, se significa y se simboliza.

De ahí que el espacio de vida cotidiana se convierta en una fuente inagotable de estudio para la Motricidad Humana como ciencia y de intervención como proceso de construcción social y personal.

Así mismo, se puede apreciar que existen ciertas disciplinas que, de una u otra forma, se acercan a estos propósitos, como cinesiología o kinesiología, la bioenergética y ciertas corrientes esotéricas. En ningún caso se pretende decir que tales disciplinas debieran o no enmendar sus caminos, sino más bien que el estudio de la motricidad humana desde la encarnación de la mente requiere adentrarse en una mirada mucho más cualitativa e interpretativa sin menospreciar lo cuantitativo, toda vez que en la fenomenología del cotidiano vivir nos encontramos precisamente con esa situación, no sólo con determinados grupos musculares o tipos de palancas, sinergias o antagonismos.

Respecto del segundo punto de la caracterización anterior, quisiéramos enfatizar la nueva dimensionalidad que adquiere la morfología y la composición de nuestra encarnación. Los elementos bioquímicos, electro físicos, morfo-funcionales y sistémico-orgánicos entran en relación con la construcción de la cognición y del lenguaje desde un punto de vista de contenido; en otras palabras, ya no es sólo el soporte o el hardware sobre el cual se cobija un software, antes bien es un componente activo, flexible y modificable que posibilita el alcance y el límite de nuestra experiencia. Tales mecanismos son inconscientes o de muy difícil acceso desde el punto de vista de nuestra voluntad, por tanto, conocer aquello que nos constituye es, sin duda, importante pero inaccesible en el momento que se configura en razonamiento, por lo menos hasta este momento. Al ser entes neurobiológicos nuestro razonamiento se hace, como proceso, no-consciente en el sentido cultural de la palabra, pero es consciente en la medida de unidad biológica y red de identidades orgánicas sin centro (Varela 2000). Tal situación implica una re-significación del ser mismo y de su 
pensamiento que tradicionalmente separa y divide incluso en aquellos que se declaran abiertamente anti dualistas.

En este orden de cosas, que la razón no pueda ser literal sino analógica y relacional, se plantea como el más identificable, pero al mismo tiempo como el más difícil de modificar, pues se adentra en lo más básico de todo conocimiento, entendiendo como conocimiento por todo aquello que da identidad a un sujeto. En este punto si compartimos que la mente no es objetiva, desapasionada y literal, inmediatamente entramos en terreno contrario, vale decir, en la subjetividad, en la pasión o mejor dicho en la emoción y, por último, en la imaginación y lo simbólico. No obstante, es aquí donde se instala la dificultad, pues hoy tenemos conocimiento minucioso de la estrecha relación entre la memoria de largo plazo y la emoción. En consecuencia, el sistema de creencias y de conocimientos que dan identidad a cada sujeto se configuran en base a la confluencia de la emoción y el afecto producidos en el devenir de determinadas situaciones, eventos o experiencias. Por tanto, están en la base de lo que llamamos persona y su cultura.

Si incluimos el lenguaje como una virtualidad de la experiencia-vivencia y la relación del signo, nos encontramos que el espacio relacional o lenguajear vamos definiendo y construyendo determinados significados que son la base de nuestra visión del mundo y obviamente de nosotros mismos.

Por ejemplo si decimos: "Hoy voy a realizar actividad física" o "mi aptitud física está deteriorada". Nos referimos en la primera frase a:

- $\quad$ Realizar algo diferente a lo cotidiano, por ende a un evento especial.

- Evento que tiene una característica determinada, a saber, una actividad física.

- El resto de la actividad del día no es física o no es fundamentalmente física.

- $\quad$ Física se refiriere a que sólo es eso o principalmente eso, o que voy a enfatizar ante todo lo físico.

- Por lo tanto, todo lo demás está por lo menos en un segundo lugar, en un tercer lugar o definitivamente no está.

En el caso de la segunda frase algunas interpretaciones podrían ser:

- Mi aptitud física está desmejorada o maltrecha y el resto de mis aptitudes están bien, o no tan mal.

- Mis aptitudes se pueden diferenciar y son identificables.

- Mi aptitud física es algo que no se mezcla con otra cosa.

Como es claro, al enunciar una determinada frase se generan diferentes posibilidades de significado y, por ende, de acción. Enmarcadas de acuerdo a la intencionalidad original del sujeto, pero que al mismo tiempo tienen una repercusión en los otros sujetos escuchantes y que están significando de acuerdo a sus propias posibilidades e historia.

El problema que quisiéramos enfatizar dice relación con que nuestro lenguaje puede facilitar o entorpecer lo que el hablante pretende significar. Mirado desde el lenguaje que en occidente se utiliza, por lo menos el español, lo que se expresa posibilita la comprensión de un sujeto que se puede dividir, separar en varias partes. Es posible que en el mismo sujeto existan partes de fácil identificación y limitación. De la misma forma, que podemos dedicarnos a una parte sin necesariamente entrar en las otras. Así mismo si una está mal, las otras no se afectan o, dicho de otra manera, podemos hacer actividades que diferencian las partes o que enfatizan una por sobre las otras. 
Surge, entonces, una pregunta de fondo a partir de lo anterior, ¿es posible tal situación en la vida cotidiana? Y por otra parte ¿cómo es que usamos un lenguaje, si es una metáfora, para referirnos a algo que sabemos que es diferente a lo que decimos?

En este contexto, es muy difícil encontrar personas del mundo de la academia que hoy por hoy se declaren definitivamente dualistas, por el contrario todos se definen o entienden que el ser humano es una integralidad, no un dogma, sino por las evidencias que la ciencia ha entregado. Tenemos un lenguaje afianzado en el dualismo cartesiano, afianzado en la experiencia de la infancia y de la juventud en un espacio relacional que potencia una situación esquizoide, al significar un sujeto dual en permanente lucha. Es una de las tareas que las disciplinas cercanas y afianzadas en la lingüística y la acción humana, debieran tomar para sí.

\section{El catalizador emocional de la encarnación}

Por último, es menester señalar que la encarnación de la mente dentro de lo que hemos llamado acoplamiento estructural con el entorno, requiere de una característica y capacidad del ser humano que le permita, precisamente, entrar en un diálogo permanente con el mismo entorno en el cual se encuentran otros seres como él-ella y distintos a él-ella, más allá de un lenguaje o condición cultural común. $O$ en caso contrario, es necesario determinar qué elementos producen en un niño la dinámica apropiada de lo que Wittgenstein denominó como juegos de lenguaje. El mismo planteó que la configuración del lenguaje se produce a raíz de intercambios lúdicos en contextos de exploración y, por sobre todo, de imitación involuntaria de los niños, aspecto que eventualmente se puede repetir cada vez que nos encontramos en ambientes ajenos o con los cuales no hay un acoplamiento fluido. La capacidad de extraer comportamientos a partir de una lectura no intencionada del entorno y de los sujetos del mismo permite un despliegue más o menos acertado o sistemas primarios de lenguaje.

Dicha capacidad se ha dado llamar como Empatía. Siguiendo a Varela (2000), podemos decir que ésta es una emoción básica que ha permitido el desarrollo y evolución del ser humano como especie. Conjuntamente Thompson $(2001,2007)$ ha realizado una categorización de la empatía desde varios puntos. Según el cual, la fenomenología actual ha ido aún más lejos en el proceso de distinción de la empatía y ha propuesto cuatro aspectos centrales del proceso de la misma que permiten analizar en forma más detallada cómo se produce el proceso de empatizar dentro de la especie humana. Estos aspectos se estructuran desde:

a. El involuntario acoplamiento o pareamiento de la corporeidad de una persona con la corporeidad de otra persona en la percepción y la acción.

b. El movimiento imaginario o transposición de mí mismo dentro de tu lugar o espacio.

c. La interpretación del tú como otro hacia mí y de mí como otro hacia el tú.

d. La percepción ética y moral del tú como una persona.

La primera dimensión de empatía, que interesa resaltar, se caracteriza desde la dimensión encarnada de nuestra existencia, que si bien es cierto es involuntaria en el sentido que no es iniciada desde un proceso de pensamiento generado desde la persona misma, sino más bien que tal proceso se genera y operacionaliza en la vinculación o ligazón entre personas con similares características corpóreas (Thompson 2007). Al decir corpóreas nos referimos a todas las dimensiones del ser humano. Tal similitud se despliega en toda la acción 
no sólo en los aspectos relacionados con la imagen o lo específicamente visual. En tal sentido, la empatía se genera en todo el actuar de la persona, en todo lo que se desprende y es observable, como en todo aquello que internaliza y no es observable o por lo menos conscientizable desde el punto de vista de la expresión verbal o del análisis explícito. Así, la empatía adquiere un estatus de percepción-acción. Varela y Thompson (2003) han expresado que tal fenómeno genera, desde el punto de vista de las neurociencias, una activación automática de áreas de la corteza cerebral acordes al comportamiento o acción que se está observando en otra persona, y con la cual se está produciendo un proceso de empatía. Tal fenómeno es de desarrollo relativamente reciente y ha sido denominado como the mirror nuerons (Rizzolatti, en Pascual 2009) (6).Vale decir, lo que hace el otro y es observado por un tercero, produce una activación de las zonas o áreas del cerebro del observador y por lo tanto de toda su corporeidad equivalentes a las de la persona observada. Esto expresa que el fenómeno de la empatía no es un ejercicio reducido a una determinada dimensión del ser humano, por el contrario es una manifestación de la integralidad sistémica del mismo. Los autores llaman a este tipo de empatía como un acoplamiento sensomotor. No obstante reconocen, como se señaló más arriba, que junto a este acoplamiento sensomotor se produce un acoplamiento afectivo o resonancia afectiva que consiste en un estado de mutua definición del estado emocional de cada uno. Desde ahí que se puede expresar, al igual que Aucouturier (2004), que la conciencia y la mente es un dialogo permanente entre lo tónico-afectivo- recursivo y lo simbólico-proyectivo.

\section{Corolario}

A partir de lo desarrollado no podemos más que concluir que el presente trabajo inaugura nuevas preguntas e inquietudes que se generan como una deriva natural de los aspectos y argumentos presentados. El primero se relaciona con preguntarnos hasta qué punto heredamos una tradición engendrada desde el dualismo cartesiano y su correspondiente traducción en la visión sobre el lenguaje tanto empirista como idealista y centralizamos nuestros esfuerzos en los aspectos periféricos creyendo que más bien nos encontramos en el centro del lenguaje. De qué nos puede servir que el ser humano sea un sujeto encarnado, acoplado con su entorno desde donde constituye mundo y se personaliza en un constate superar de sus debilidades y carencias. Una eventual respuesta se orientaría, desde nuestra opinión, a configurar nuevos lenguajes, cercanos y vigilantes de la experiencia desplegada, más que desde la tradición normativa.

Adicionalmente, poco nos serviría dentro de la intención hasta aquí desplegada centralizarnos en algún o algunos aspectos descritos más arriba pues de alguna manera caeríamos en una parcialidad argumentativa intentando expresar lo contrario. Por lo tanto, la encarnación del lenguaje y de su máxima expresión como es la vida o experiencia mental, es mucho más que una mirada reivindicativa de lo que tradicionalmente se ha llamado cuerpo, más bien es una redefinición antropológica y por tanto epistemológica, con sus respectivas resonancias en todas las dimensiones del actuar. Es un alerta de conciencia a una experiencia y lenguaje más pleno, es asumir que la imposibilidad del no comunicar que nos presentara Waztlawick en los años ochenta, se genera en la comprensión que nuestra motricidad en todas sus posibles manifestaciones constituye el texto y registro de nuestra existencia tanto personal como social y por consecuencia cultural.

El vals de Benedetti nos invita desde la honestidad de la inspiración e intuición poética a reconocernos como tal. La misma invitación nos hacen desde la vanguardia investigativa en Inteligencia Artificial, las neurociencias y por sobre todo la tradición fenomenológica que no pretende otra cosa (esta última) que dar cuenta comprensivamente de la experiencia y acción humana, es decir, es más que un meta-dialogo o dicho desde el contexto de este trabajo, es una meta-acción. 
Esta aproximación puede permitirnos desarrollar un lenguaje más unitario y coherente en función de las diferentes dimensiones del lenguaje, por ejemplo situar diferentes manifestaciones estéticas, lúdicas, ergonómicas, cotidianas como la danza o baile. La búsqueda de tales expresiones culturales por diferentes grupos sociales y etarios podría deberse a la búsqueda de nuevos relatos, nuevos textos, posibilidades de ampliar, profundizar o alterar el texto personal. De igual forma los movimientos sociales, dentro de una sociedad, también expresan discursos. Quizás esto es obvio, pero ¿qué ocurre con aquellos lenguajes no verbales, con la prosodia, la respiración, las actitudes y disposiciones en tanto corporeidades en red o entramados desde diálogos no logarítmicos, sino más bien cada vez más analógicos, metafóricos y gestuales?

¿Qué es aquello que se comprende desde las acciones que emprendemos en la cotidianidad de nuestra vida social? Esa misma cotidianidad nos impulsa a trascender lo verbal, a enfatizar y focalizarnos sobre los desempeños recurrentes fortalecidos por las costumbres, las normas y los condicionamientos. Tal derrotero nos puede permitir avanzar un poco más hacia la comprensión del fenómeno humano desde su propio lenguaje. Es un punto interesante que se puede analizar en virtud de las habitudes y su impacto en la interpretación de sí mismo y de la cultura en la cual se vive. Un análisis crítico del discurso, si consideramos la motricidad como texto, podría traernos una aproximación de las relaciones y marcos de poder, pero situado en un nivel que se transversaliza no solo en lo textual y semántico, sino por sobre todo en lo pragmático y en la relacionalidad del tejido social. Esperamos más adelante adentrarnos en tales desafíos.

\section{Notas}

(1) Este trabajo se realiza en el contexto del Proyecto de Investigación Fondecyt № 11080297: Corporeidad y Aprendizaje en el Contexto de la Enseñanza General Básica: Comprensión y Descripción de los Procesos de Construcción de Conocimiento desde la Acción Relacional de los Actores.

(2) Rene Descartes publicó en 1637 su Discurso del Método. En tal obra aparece la famosa frase "Pienso luego existo", de ella se desprende que lo sustantivo de la existencia humana se centraliza en la razón o pensamiento, por lo tanto la existencia, en un sentido amplio y profundo, se centra en la razón y no el cuerpo. La mente, entonces, como espacialidad configurativa de la razón, debiera posibilitar y controlar la corporalidad del sujeto.

(3) No obstante para una mayor profundización de la temática se siguiere revisar Varela (1990). En dicha obra se presenta un exhaustivo análisis de la evolución de las diferentes teorías como de traducción en el proceso de conocer en la cotidianidad.

(4) En este sentido cabe recordar que la ciencia se ha nutrido y desarrollado bajo una visión mecanicista del mundo propia de la interpretación newtoniana de lo que se entiende por realidad (Berman 1998). Este enfoque permitió una apertura hacia la manipulación e intervención de todo lo que se definiese como natural. Es así como el desarrollo de la medicina desde lo que Manuel Sergio (2007) define como enfoque biomédico favoreció la experimentación en laboratorios o en vitro, con diferentes especies y entornos. De igual forma posibilitó una comprensión de todo lo natural como ajeno a lo racional, por lo tanto susceptible de ser manipulado y dominado en función de lo racional y mecánico. Berman (1998) sostiene que tal argumento sirvió en muchos casos para fundamentar el esfuerzo colonizador, como también para asentar la estructura social dominante y un tipo de lenguaje que sostenía y potenciaba el status quo. 
(5) Warhnemen, palabra alemana para percepción. Sich bewegen, palabra alemana traducida al español como movimentar-se.

(6) Las neuronas espejos como desarrollo explicativo sostiene que existen grupos de neuronas ubicadas en la zona pre-motora del cortex cerebral que se activan tanto en la acción propia del chimpancé que se encontraba en el experimento y que ocurría el mismo fenómeno cuando el chimpancé observaba en otro sujeto la acción. Tal proceso se producía por lo menos seis actos motores que incluyen agarrar con el brazo y la boca, agarrar con la mano, asegurar, arrancar, esperar y llevar a la boca. Para el Rizzolatti estas acciones constituyen un "vocabulario neuronal" que a su vez se correlacionan tanto con sus actos propiamente tal como con sus interpretaciones, por lo tanto pueden ser utilizados tanto para repetir acciones como para comprender. De tal forma las neuronas espejos podrían intervenir en los procesos de visaje o reconocimiento de las acciones de otro individuo, diferenciándolas de otras acciones y utilizarlas para adaptar el comportamiento en consecuencia. En palabras de J.P. Changeux "es entonces plausible suponer que estas neuronas espejos participan en la comunicación inferencial de las intenciones del otro observado" (2003: 151).

\section{Bibliografía}

Aucouturier, B. 2004. Los fantasmas de la acción. Barcelona: Grao Ediciones.

Austin, J. 1982. Cómo hacer cosas con palabras. Barcelona: Paidos.

Bachelard, G. 1997. La formación del espíritu científico. Barcelona: Siglo XXI.

Benedetti, M. 1993. Inventario II. Poesía 1986-1991. Barcelona: Visor.

Berman, M. 1998. Cuerpo y espíritu. La historia oculta de occidente. Santiago: Cuatro Vientos.

Damasio. A. 2000. Sentir lo que sucede. Santiago: Andrés Bello

Changeux, J.P. 2003. A verdade e o Cérebro. O homen de verdade. Lisboa: Instituto Piaget.

Gabler, H. 1996. Einführung in die Sportpsychology. Schondorft: Hoffmann.

Gadamer, H.G. 1977. Verdad y método I. Salamanca: Sígueme.

Gallagher, S. 2006. How the body shapes the mind. New York: Oxford University Press. Gallagher, S. y Zahavi, D. 2008. The phenomenological mind: an introduction to philosophy of mind and cognitive science. New York: Routledge.

Heisemberg, W. 1981. A imagen da natureza na física moderna. Lisboa: Libros de Brasil.

Husserl, E. 1949. Ideas. Relativas a una fenomenología pura y una filosofía fenomenológica. Madrid: Fondo de la Cultura Económica.

Lakoff, G. y Johnson, M. 1999. Philosophy in the flesh the embodied mind and its challenge to western thought. New York: Basic Books.

Sergio, M. 2007. Algunas miradas sobre el cuerpo. Popayán: Universidad del Cauca. 
Merleau-Ponty, M. 2000. Fenomenología de la percepción. Madrid: Península

Morin, E.2003. El método. La humanidad de la humanidad, la identidad humana. Madrid: Cátedra.

Noe, A. 2004. Action in perception. Cambridge: MIT Press.

Pascual, R. 2009. Neurobiología del self y sus extravíos. Aproximación ontogénica y biosocial. Valparaíso: Ediciones Universitarias de Valparaíso.

Pfeifer, R. y Bongard, J. 2007. How the body shapes the way we think. Massachusetts: MIT Press.

Prigogine, I. 1997. ¿Tan sólo una ilusión? Una exploración del caos al orden. Barcelona: Tusquets Editores.

Schütz, A. 1993. La construcción significativa del mundo social. Introducción a la sociología comprensiva. Barcelona: Paidós Básica.

Thompson, E. 2007. Mind in life. Biology, phenomenology and the sciences of mind. Massachusetts: Harvard University Press.

Thompson, E. 2001. Between ourselves: Second-person issues in the study of consciousness. Thorverton, UK: Imprint Academic.

Toro, S. 2006. Conocimiento y motricidad, aproximaciones y desafíos. Revista Pensamiento Educativo 38: 6274.

Toro, S. 2002. Psicología de la acción. Perfiles Siglo XXI (5): 70-82.

Varela, F. 2000. El fenómeno de la vida. Santiago: Dolmen.

Varela, F. 1990. Conocer. Barcelona: Gedisa.

Recibido el 27 Oct 2009

Aceptado el 4 Feb 2010 